\title{
Multivariable feedback control of nuclear reactors
}

\author{
RUNE MOEN $\dagger$
}

Keywords: nuclear reactor, multivariable feedback control, optimal control.

\begin{abstract}
Multivariable feedback control has been adapted for optimal control of the spatial power distribution in nuclear reactor cores. Two design techniques, based on the theory of automatic control, were developed: the State Variable Feedback (SVF) is an application of the linear optimal control theory, and the Multivariable Frequency Response (MFR) is based on a generalization of the traditional frequency response approach to control system design.

The two design methods lead to different structures for the control system, and both were studied in simulation. The objective of the paper is to show the benefits and problems related to the SVF and MFR techniques, when used to design a control system for a typical pressurized light water reactor core. The models and the design procedures are described and results from simulation experiments are presented.

The conclusion of the simulation experiments is that the two methods SVF and MFR are similar with respect to performance and computational requirements. However, during the design of this particular control system, the MFR method was easier to apply. The SVF control system includes a Kalman filter state estimator. The simulation experiments show that the state estimator converges sufficiently fast for use with the SVF control system.
\end{abstract}

\section{Introduction}

In a large nuclear power plant strict requirements are put to the spatial power distribution of the reactor core. Oscillations are easily excited if the total power is changed, i.e. if daily load cycle operation is desired (that is full power operation during the day, and reduced power during the night).

The oscillations are caused by the dynamic effect of xenon and iodine. Xenon is an extremely good neutron absorber which is produced from fission directly and through decay of iodine (half-life of $6.7 \mathrm{~h}$ ) and removed through decay (half-life of $9 \cdot 2 \mathrm{~h}$ ) and absorption. Iodine in turn comes from fission. This dynamic process leads to power oscillations initiated by power changes, and with a period of 20 to $30 \mathrm{~h}$. Of course the regulating system of the reactor manages to control the power to the setpoints, but to achieve this control rods which usually disturb the spatial power distribution has to be used. This initiates the power distribution oscillation caused by the same xenon-iodine dynamics, shifting a power peak between the upper core half and the lower half.

Several regulating system designs exist to minimize this spatial power oscillation. At the Halden Reactor Project two classes of power distribution control methods have been studied. One is a predictive deterministic optimal control method named Multistage Mathematical Programming. This method has been presented in MIC

Received 28 March 1982

† Institutt for Energiteknikk, OECD Halden Reactor Project, Halden, Norway. 
1980, vol. 1, No. 2. The article describes in more detail the nuclear reactor core dynamics and the core model design.

The other class is use of multivariable feedback methods for control of the power distribution to some specified setpoint. Formulation of the feedback control problem has been made both in the time and frequency domain, and a comparison of the two formulations is presented in this paper. The two methods are named State Variable Feedback (SVF) and Multivariable Feedback Response (MFR) respectively. SVF is based on the Linear Optimal Control and Estimation Theory, while MFR is an Output Feedback method utilizing the multivariable extension of traditional frequency domain techniques of Nyquist and Bode, mainly based on Rosenbrock (1974).

\section{Problem description}

\subsection{The plant}

For this investigation, one class of nuclear reactors has been studied, that is the Pressurized Light Water Reactor. The released nuclear energy heats up the fuel pins which are cooled by water under high pressure. The coolant which flows along the fuel pins from the bottom of the core upwards, also acts as a neutron moderator slowing down the speed of the neutrons to generate new fissions in the fuel. The nuclear power generated depends on the atomic composition and temperatures of the core, which change continuously during power operation of the reactor. Soluble boric acid which is a strong neutron absorber may be mixed into the coolant water, causing the nuclear power to be reduced almost uniformly in the reactor core. The higher concentration the more reduction of power. Another type of power controller is the control rod banks which are rods of highly neutron-absorbing material entered from the above into the core. The control rods reduce the power in their surrounding, and depending on the insertion depth, can be used for changing both the total power and the power distribution in the core.

The Nuclear Steam Supply System (NSSS) of the power plant is schematically drawn in Fig. 1. From the reactor core the coolant water is piped to the steam generators where the heat in the high-pressure water is transferred to steam in the secondary or turbine coolant circuit. The steam generated runs the turbines which in turn drive the electric generators of the plant. The secondary circuit is closed by pumping the condensed steam back to the steam generators, and the primary coolant circuit is closed by pumping the cooled water to the reactor core.

The nuclear power is usually controlled by the control rod insertion and the boric acid solution concentration. In addition the coolant temperature of the primary circuit influences the power: usually increased temperature reduces the power. The temperature may not be controlled directly, but will depend on the nuclear power and the turbine power production.

The control system of the reactor which of course only is a part of the nuclear power plant control system regulates the control rod insertion and the boric acid solution concentration of the primary coolant. The control rods of this particular reactor are regulated automatically by the Reactor Regulating System (RRS) to control the primary coolant temperature to a power-dependent setpoint. The sequence in which the control rods are moved is predetermined in order to minimize the distortion of the ideal spatial power distribution of the reactor core. 


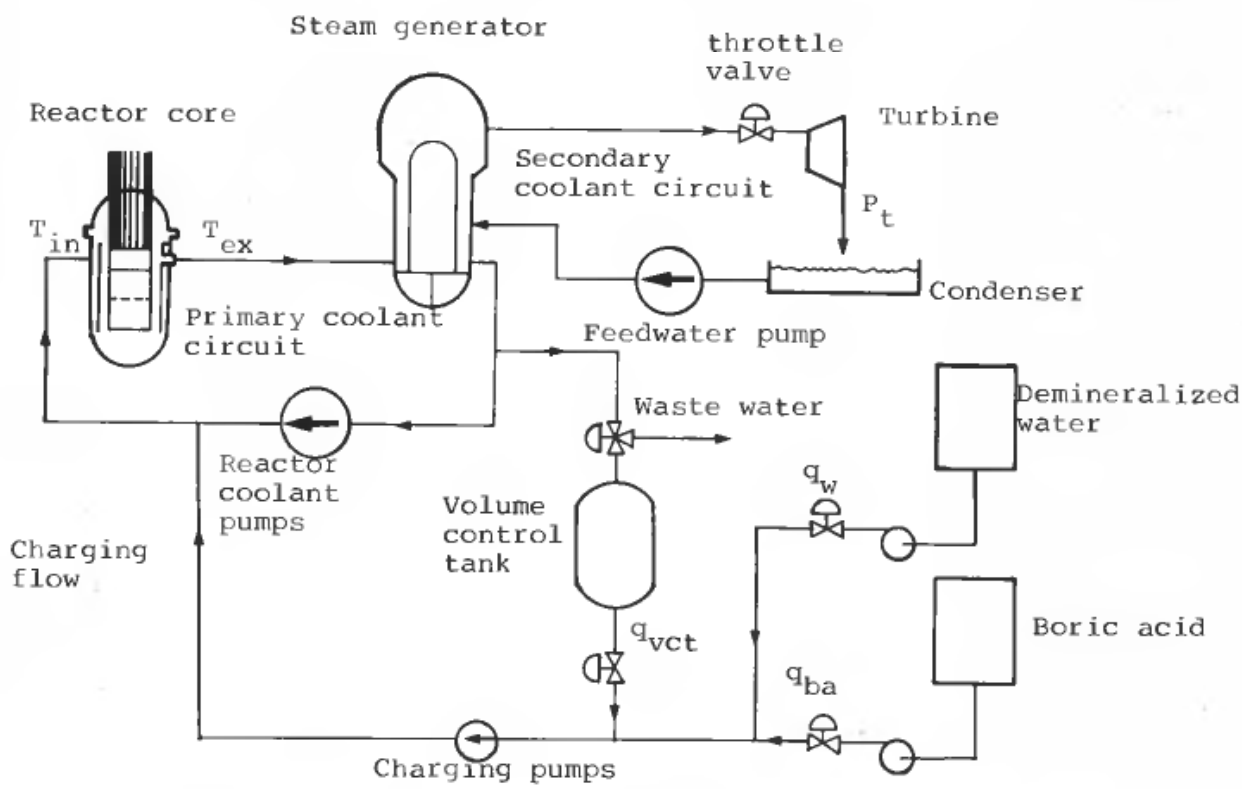

Figure 1. The nuclear steam supply system of a Pressurized Light Water Reactor power plant (schematically).

However, the non-linear effect of the control rods cannot be avoided. Firstly the reactivity change is concentrated around the rod tip, so a small movement of the rod causes a big change in reactivity, but only when the rod tip passes. Secondly, as a consequence, when the first rod bank starts the insertion from the top of the core, the bottom core power is not influenced and the axial power distribution becomes more and more skew. Only after the first rod bank is $60 \%$ inserted and the second rod bank inserts, the spatial power distribution is not seriously disturbed by the control rods.

The boron concentration is controlled by the Chemical and Volume Control System (CVCS) which is manually operated. Reactor coolant is continually injected to the primary circuit from the CVCS at constant rate and with a boron concentration determined by the CVCS operation. Usually most of the fluid comes from the Volume Control Tank (VCT), but the boron concentration of the injected fluid may be changed by mixing in demineralized water or high concentration boric acid through the control valves. Due to the length of the connecting pipes there is a delay $T_{\mathrm{d}}$ (approximately 4 minutes) before the charging flow reached the primary circuit after leaving the blending point in the CVCS.

The injection of fluid generates waste coolants which have to be demineralized. This chemical process is relatively expensive and one tries to avoid it by operating the reactor within minimum variation of boric acid concentration.

\subsection{Control system objective}

The objective of this work is to study the performance of multivariable feedback control, and to compare the state feedback method and the output feedback method. The main variables to be controlled are the average power and the power distribution in the reactor core. 
The nuclear power must follow the turbine power in such a way that the moderator average temperature $T_{\text {avg }}$ is kept at a given value, dependent of the turbine power ( $T_{\text {avg }}$ programme). A suitable process output is therefore the core average temperature $T_{\text {avg }}$ which is strongly connected to the core power. The second process output is the Axial Shape Index (ASI) which represents the axial power distribution. The ASI is defined as power in the bottom core half minus power in the top half divided by total core power. ASI has to be kept within a control band about a given constant value.

A typical disturbance is the turbine power variation. One important case of power variation is the daily load cycle, where power is reduced from $100 \%$ of rated power to a lower value, say $50 \%$ or $75 \%$, for a period of $6-8$ hours after which the power is returned to $100 \%$. Other cases of variation may be return to full power after sudden power drops.

The controllers are the control rod positions and the boric acid concentration of the coolant. The boron concentration cannot be controlled directly, but the rate of change may be controlled by commands to the CVCS. The boric acid concentration of the charging flow may vary from $0 \mathrm{ppm}$ to $4000 \mathrm{ppm}$, depending on the mixture of $q_{\mathrm{vct}}, q_{\mathrm{w}}$, and $q_{\mathrm{ba}}$ (Fig. 1). Since the primary coolant boron concentration varies from around $700 \mathrm{ppm}$ to about $20 \mathrm{ppm}$, dilution of boron is much slower than boration. This dilution rate limitation, as well as the time delay of the charging pipes seriously reduce the effectiveness of boron as a reactivity controller.

The task of control systems is to keep $T_{\text {avg }}$ and ASI to their setpoints, using CEA movements and charged boric acid concentration, simultaneously, for control. The objective is to maintain control during reasonable disturbances. The selected inputs represent the time derivatives of the control rod position and the boric acid concentration of the primary coolant.

Note that the control systems are designed as regulators, i.e., the main requirements are stability in all operating conditions, as opposed to servo systems which are designed to meet closed loop response requirements during rapid changes in the setpoints.

\section{State variable feedback (SVF)}

\subsection{Model description}

The State Variable Feedback design technique for multivariable control systems is based on the well established Linear Optimal Control and Estimation Theory. The process is described in terms of a linear time invariant dynamic system:

$$
\left.\begin{array}{l}
\dot{x}=A x+B u+C_{\mathrm{x}} d+C_{\mathrm{m}} v \\
y=D x \\
y_{\mathrm{m}}=D_{\mathrm{m}} x+w
\end{array}\right\}
$$

where $\boldsymbol{x}$ is the state vector, $\boldsymbol{y}$ is the output vector, $\boldsymbol{u}$ is the control vector and $\boldsymbol{d}$ is the disturbance vector. $A, B, C_{\mathrm{x}}$ and $D$ are matrices, computed by the TOPO programme that model the system behaviour. The state variables in $\boldsymbol{x}$ are

$$
\begin{aligned}
& x_{1}=\text { inlet temperature of primary coolant } \\
& x_{2}=\text { exit temperature of primary coolant }
\end{aligned}
$$


$x_{3}=$ average temperature of secondary coolant

$x_{4}=$ CEA position

$x_{5}=$ boric acid concentration of primary coolant

$x_{6}=$ average xenon concentration of lower core half

$x_{7}=$ average xenon concentration of upper core half

$x_{\mathbf{8}}=$ average iodine concentration of lower core half

$x_{9}=$ average iodine concentration of upper core half

The system is assumed to be subject to noise, and for the design $v$ and $w$ are assumed to be noise vectors for process and measurement noise. $C_{\mathrm{m}}$ is the process noise coupling matrix, and $D_{\mathrm{m}}$ is the measurement matrix. $y_{\mathrm{m}}$ is the measurement vector, different from the output $y$.

As described in 2.2, the output vector contains primary coolant average temperature $T_{\text {avg }}$ and Axial Shape Index, ASI. The boric acid concentration at the charging pump, and the control rod speed make up the control input vector. The disturbance vector is the turbine power $\boldsymbol{P}_{\mathbf{t}}$. Note that boric acid concentration of the primary coolant and control rod position are states, present in the state vector, i.e., the system already contains an integral action.

The state vector is not directly measurable, because it contains the xenon and iodine concentration as state variables. These variables need to be estimated or calculated from the core power history. For the estimation we assume the following output $y_{\mathrm{m}}$ to be measurable:

$$
\begin{array}{ll}
y_{\mathrm{m}, 1}=\text { Lower core half power density } & P_{1} \\
y_{\mathrm{m}, 2}=\text { Upper core half power density } & P_{\mathrm{u}} \\
y_{\mathrm{m}, 3}=\text { Coolant inlet temperature } & T_{\mathrm{in}} \\
y_{\mathrm{m}, 4}=\text { Coolant exit temperature } & T_{\mathrm{ex}} \\
y_{\mathrm{m}, 5}=\text { CEA position } & P_{\mathrm{r}} \\
y_{\mathrm{m}, 6}=\text { Coolant boric acid concentration } P_{\mathrm{c}}
\end{array}
$$

The measurement of $P_{\mathrm{c}}$ is doubtful, so the effect of deleting the boron concentration measurement had to be examined. The CEA position may be measured with great accuracy, while the core power is subject to uncertainty.

The theory requires that the process noise vector $v$ represents white uncorrelated noise with zero mean. This is however, not the case for this system, where the process noise rather describes the deviation from the linear model. Applying the separation theorem in this case, obviously violates the optimality condition. This is however, not serious for the solution of the control problem, because the main objective of the controller is to stabilize the xenon-induced oscillations.

For the design of the state feedback the turbine power disturbance $\boldsymbol{d}$ may be included in the state, and the noise sources $v$ and $w$ may be omitted. This results in an augmented state vector and a corresponding reformulation of the system matrices in (1) giving the deterministic state equation.

$$
\left.\begin{array}{l}
\dot{\tilde{x}}=\tilde{A} \tilde{\boldsymbol{x}}+\tilde{B} \tilde{u} \\
\boldsymbol{y}=\tilde{D} \tilde{\boldsymbol{x}}
\end{array}\right\}
$$


For the estimation problem an other rearrangement is performed, including the disturbance $\boldsymbol{d}$ into the control input $\boldsymbol{u}$, yielding a measurement model.

$$
\left.\begin{array}{l}
\dot{x}_{\mathrm{m}}=A x_{\mathrm{m}}+B_{\mathrm{m}} u_{\mathrm{m}}-C_{\mathrm{m}} v \\
y_{\mathrm{m}}=D_{\mathrm{m}} x_{\mathrm{m}}+w
\end{array}\right\}
$$

The vectors $x, x_{m}, u, u_{m}$, and $y$ expresses in eqns. (2) and (3) the deviation from the linearization state $x_{0}, u_{0}, y_{0}$, and $d_{0}$.

\subsection{Control problem formulation}

The problem is stated as that of finding the vector $\tilde{\boldsymbol{u}}$ which minimizes the following performance index

$$
J=\int_{t_{\mathrm{o}}}^{\alpha}\left[\tilde{y}^{\mathrm{T}} Q_{\mathrm{y}} \tilde{y}+\tilde{x}^{\mathrm{T}} \tilde{Q}_{\mathrm{x}} \tilde{\boldsymbol{x}}+\tilde{u}^{\mathrm{T}} R \tilde{u}\right] d t
$$

subject to the constraints given in eqn. (2) and with initial condition $\tilde{\boldsymbol{x}}\left(t_{0}\right)$ given. For convenience the continuous time version is discussed here, but the corresponding discrete form was actually programmed in the SVF algorithm. The solution of the control problem results in a control law of the form

$$
\tilde{\boldsymbol{u}}=G \tilde{\boldsymbol{x}}
$$

where $\tilde{\boldsymbol{x}}$ is the actual or estimated augmented state and $\tilde{\boldsymbol{u}}$ is the control input.

To show the structure of the resulting control system, we reformulate eqn. (4) by using the condition $\tilde{\boldsymbol{y}}=D \tilde{\boldsymbol{x}}$ and splitting the vectors.

$$
\begin{array}{r}
J=\int_{t_{0}}^{\infty}\left[\left(x-x_{0}\right)^{\mathrm{T}}\left[D^{\mathrm{T}} Q_{\mathrm{y}} D+Q_{\mathrm{x}}\right]\left(x-x_{0}\right)+\left(d-d_{0}\right)^{\mathrm{T}} Q_{\mathrm{d}}\left(d-d_{0}\right)\right. \\
\left.+\left(u-u_{0}\right)^{\mathrm{T}} R\left(u-u_{0}\right)\right] d t
\end{array}
$$

The corresponding feedback control law

$$
u=u_{\text {set }}+G_{\mathrm{x}}\left(x-x_{\text {set }}\right)+G_{\mathrm{d}}\left(d-d_{0}\right)
$$

is described in Fig. 2. The 'state setpoint' $\boldsymbol{x}_{\text {set }}$ and 'control input setpoint' $\boldsymbol{u}_{\text {set }}$ are computed from the setpoint $\boldsymbol{y}_{\text {set }}$ using the steady state equation

$$
\left.\begin{array}{rl}
0 & =A x_{\text {set }}+B u_{\text {set }} \\
y_{\text {set }} & =D x_{\text {set }}
\end{array}\right\}
$$

assuming the disturbance $\boldsymbol{d}$ equal zero and the output setpoint $\boldsymbol{y}_{\text {set }}$ known. This results in a control system with feedback from the estimated (or actual) state vector $\boldsymbol{x}$, and with feedforward from the turbine power disturbance $\boldsymbol{d}$.

The state estimation problem is solved from the system description eqn. (3) and assuming the process noise $C_{\mathrm{m}} v$ representing the linear model uncertainty and $w$ representing the measurement noise.

The Kalman filter calculation gives the gain matrix $R_{\mathrm{k}}$ of the optimal estimator described by the iterative estimation equation

$$
\hat{\mathbf{x}}(k)=\hat{\mathbf{x}}(k \mid k-1)+R_{\mathrm{k}}\left[y_{\mathrm{m}}(k)-D_{\mathrm{m}} \hat{x}(k \mid k-1)\right]
$$




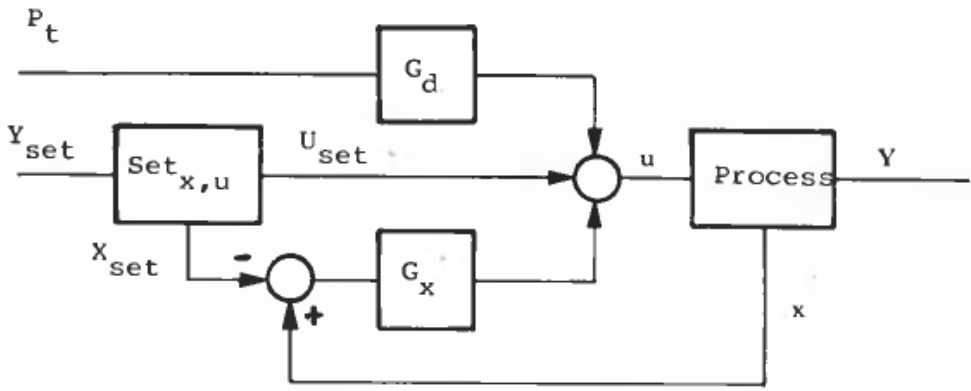

Figure 2. Structure of the SVF regulator with feedback control from state and with feedforward control from disturbances.

where $\hat{x}(k)$ is the estimated state at step $k$, and the predicted estimate $\hat{x}(k \mid k-1)$ is given by

$$
\hat{x}(k \mid k-1)=\Phi \hat{x}(k-1)+\Delta u_{\mathrm{m}}(k-1)
$$

$\Phi$ and $\Delta$ are the transition and transmission matrix, respectively

$$
\left.\begin{array}{l}
\Phi=e^{\mathrm{A} T} \\
\Delta=\left(\int_{0}^{T} e^{\mathrm{A} \tau} d \tau\right) \cdot B_{\mathrm{m}}
\end{array}\right\}
$$

where $T$ is the sampling step length and $A$ and $B$ the system and control matrix in eqn. (3). The complete SVF regulator consists of a cascading of the optimal estimator with a deterministic optimum controller. The filter gain matrix $R_{\mathrm{k}}$, the feedback matrix $G_{\mathrm{x}}$, and the disturbance feedforward matrix $G_{\mathrm{d}}$ are all computed by the MULTEC computer programme.

\subsection{Simulated test case}

Some test cases have been simulated to study the performance of both the SVF and MFR regulators. The daily load cycle case is of special interest, both because it is a likely operating strategy and it requires proper co-ordination of the controllers for good performance. Another interesting case is stabilization of the reactor power and power distribution after disturbances.

The simulated test plant is the Combustion Engineering System 80 nuclear power plant, as described in $\S 2.1$. The simulation is performed by the non-linear TOPO model, which also is utilized for the generation of linear control models.

The simulated daily load cycle is indicated in Fig. 3. The load sequence starts with a decrease in power over $2 \mathrm{~h}$ from full power at equilibrium to a constant low power level $(50 \%, 60 \%$ or $75 \%)$. The lower power remains for $6 \mathrm{~h}$, then full power is restored in $2 \mathrm{~h}$. To complete a $24 \mathrm{~h}$ cycle, the power is then kept at $100 \%$ for the remaining $14 \mathrm{~h}$. During the load cycle, $T_{\text {avg }}$ is requested to follow a power-dependent programme, while ASI has to be kept close to the initial value.

Several simulation experiments have been performed to analyse the sensitivity of the performance index parameters in eqn. (6) and in the simulations presented here the control system is in some respect tuned for the $75 \%$ loadcycle case. Figures 4,5 , and 6 give the simulation results for the SVF controlled $75 \%$ loadcycle, $60 \%$ loadcycle, and $50 \%$ loadcycle. 


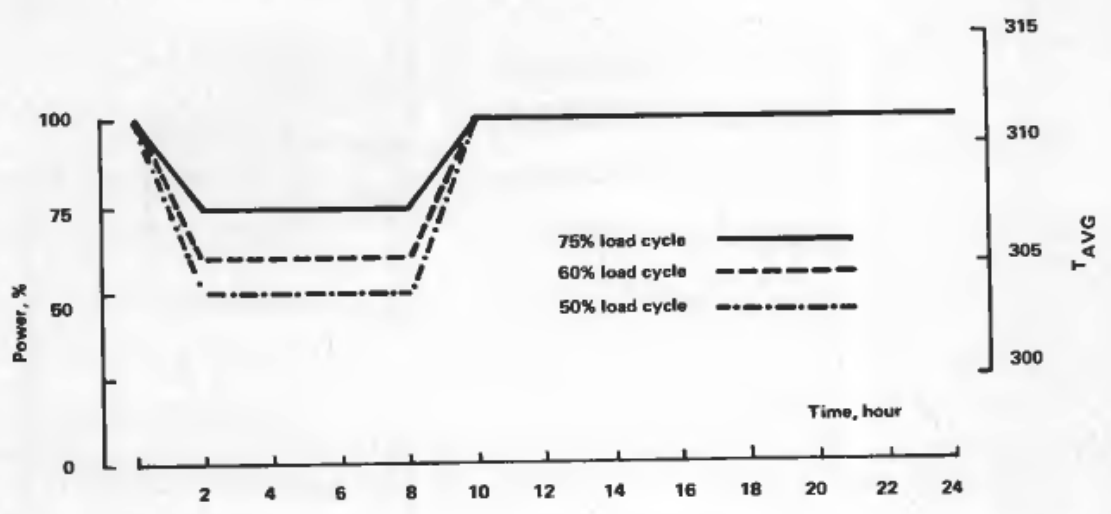

Figure 3. Power load cycling during a $24 \mathrm{~h}$ period. The related average temperature is indicated.

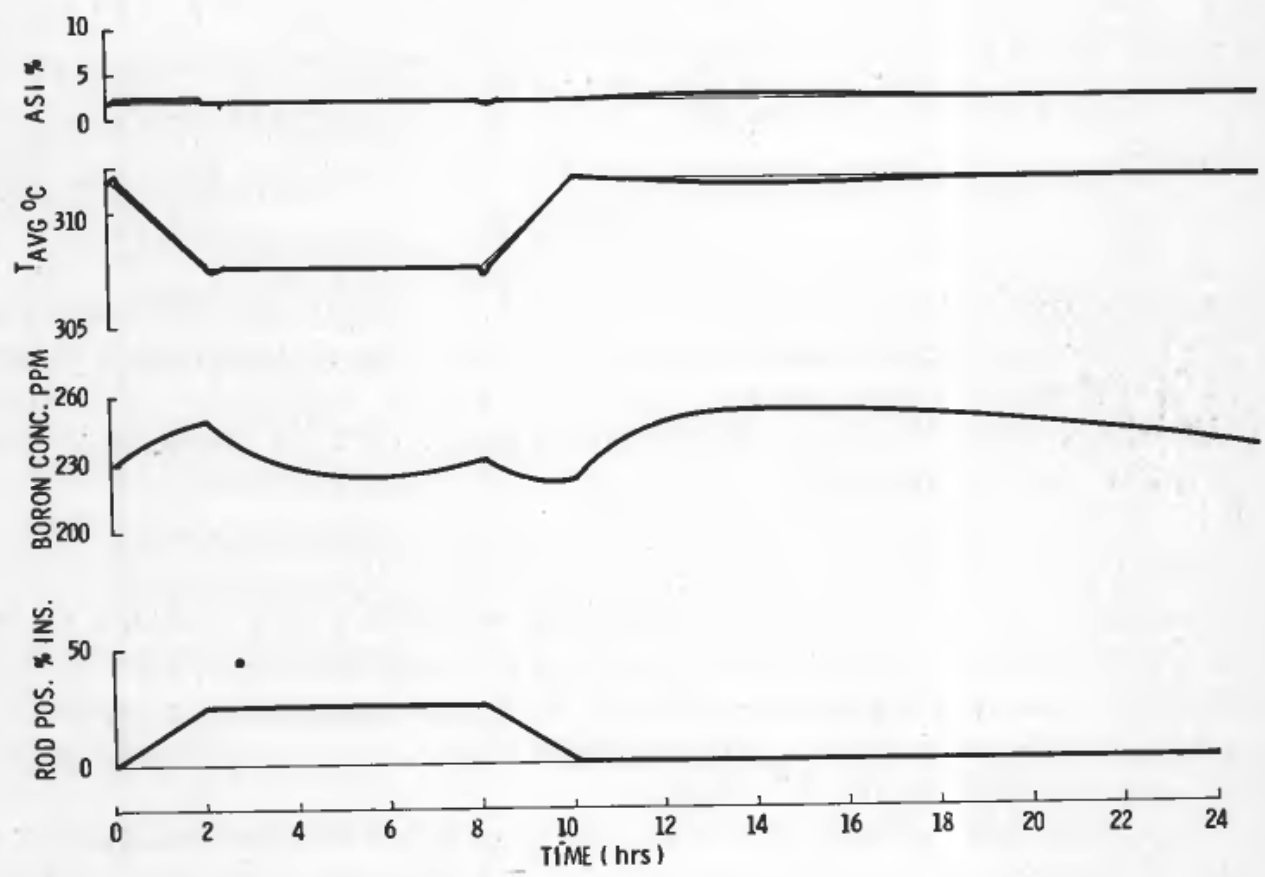

Figure 4. SVF controlled $75 \%$ load cycle.

As may be seen from Fig. 4 the $75 \%$ loadcycle is well controlled by the SVF control system. Both $T_{\text {avg }}$ and ASI are kept within a narrow band around the setpoints. However, the optimality condition of constant setpoint is not present, so one does not expect this control law to be the best one for the daily loadcycle case.

The optimality condition is even more violated in Fig. 5 with the $60 \%$ loadcycle. The boron control saturates as soon as $60 \%$ power is reached after $2 \mathrm{~h}$. The xenon concentration increases so rapidly that the CVCS charging pump cannot provide enough demineralized water to compensate for the reactivity decrease. This is a hard constraint which is not taken into consideration during SVF design, and the result is a power mismatch between turbine and core causing reduced temperature 


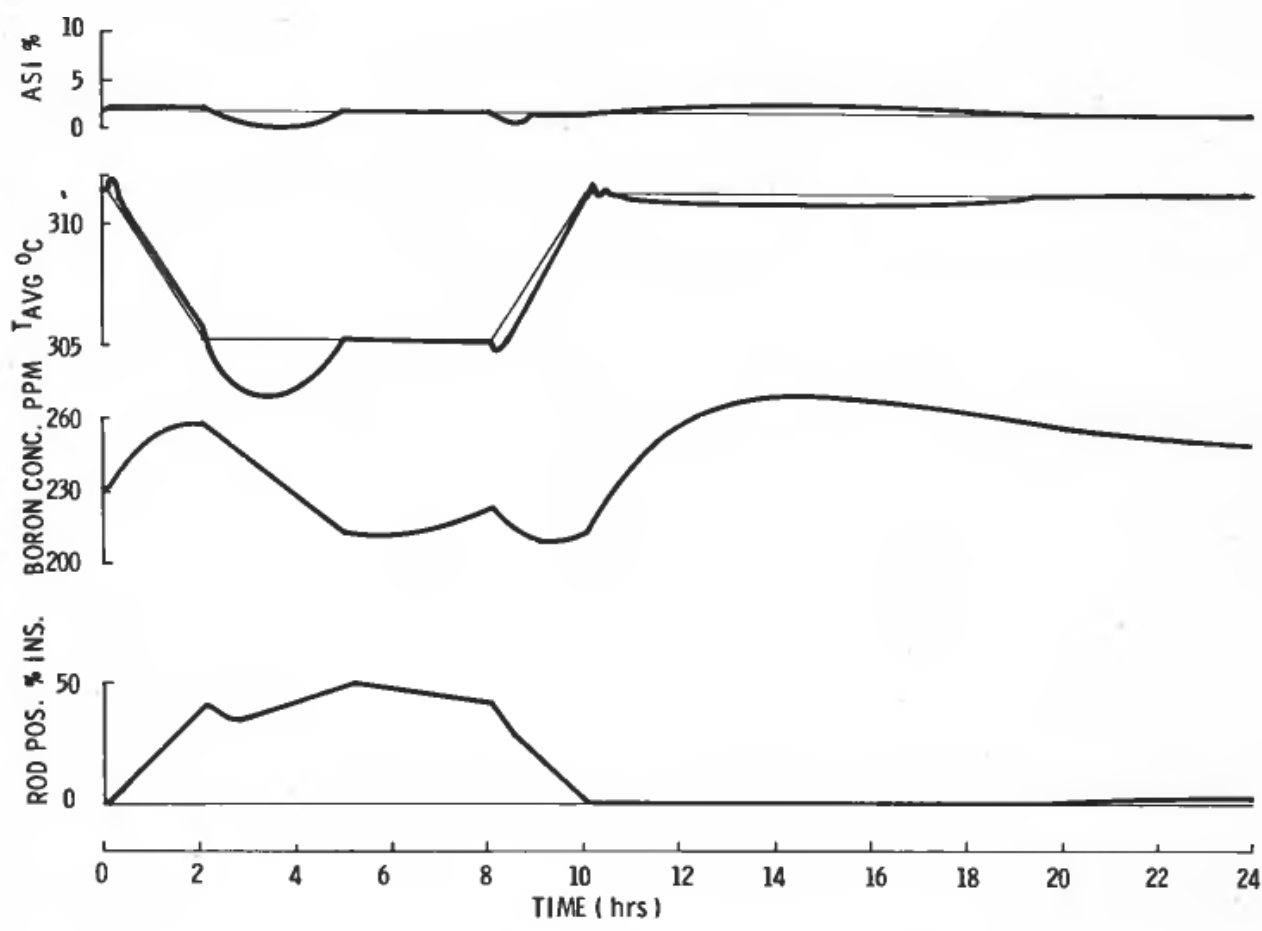

Figure 5. SVF controlled $60 \%$ load cycie.

for $3 \mathrm{~h}$. After $10 \mathrm{~h}$ the return to full power is completed, but the control rods are fully withdrawn and this saturation causes a small $T_{\text {avg }}$ and ASI deviation which cannot be controlled.

A $50 \%$ loadcycle causes significant deviation from setpoint over long periods. The boron saturation is present during the decreased power period, and the related $T_{\text {avg }}$ and ASI deviation is observed in Fig. 6. The non-linear effect of the control rods may be observed. Significant steps are seen in the simulated response when the control rods are about $50-60 \%$ and $110-120 \%$ inserted after 2 and $6 \mathrm{~h}$ respectively.

In all the simulation experiments, feedback was taken from the estimated state. To check the state estimator quality, the effect of using estimated state values was tested by taking the feedback from actual states instead of estimated states. Figure 7 presents the control performance given as deviation from setpoints for the $75 \%$ loadcycle case. From the result it was concluded that the estimation error is negligible from the control performance point of view.

The effect of the boron charging time delay of $4 \mathrm{~min}$ was also studied. Simulations were performed to compare the control performance both with and without the CVCS time delay included. Note that the controller was designed assuming that no time delay exists. The results, given in Fig. 8 show that the control is not much influenced by introducing the time delay. The time delay is so small compared to the period of the xenon oscillation that xenon stability is not affected.

The SVF controller is actually designed more for situations where the controller is used tosstabilize the reactor core after a disturbance initiating xenon oscillation. Simulations have been performed to study the control after a disturbance in which 


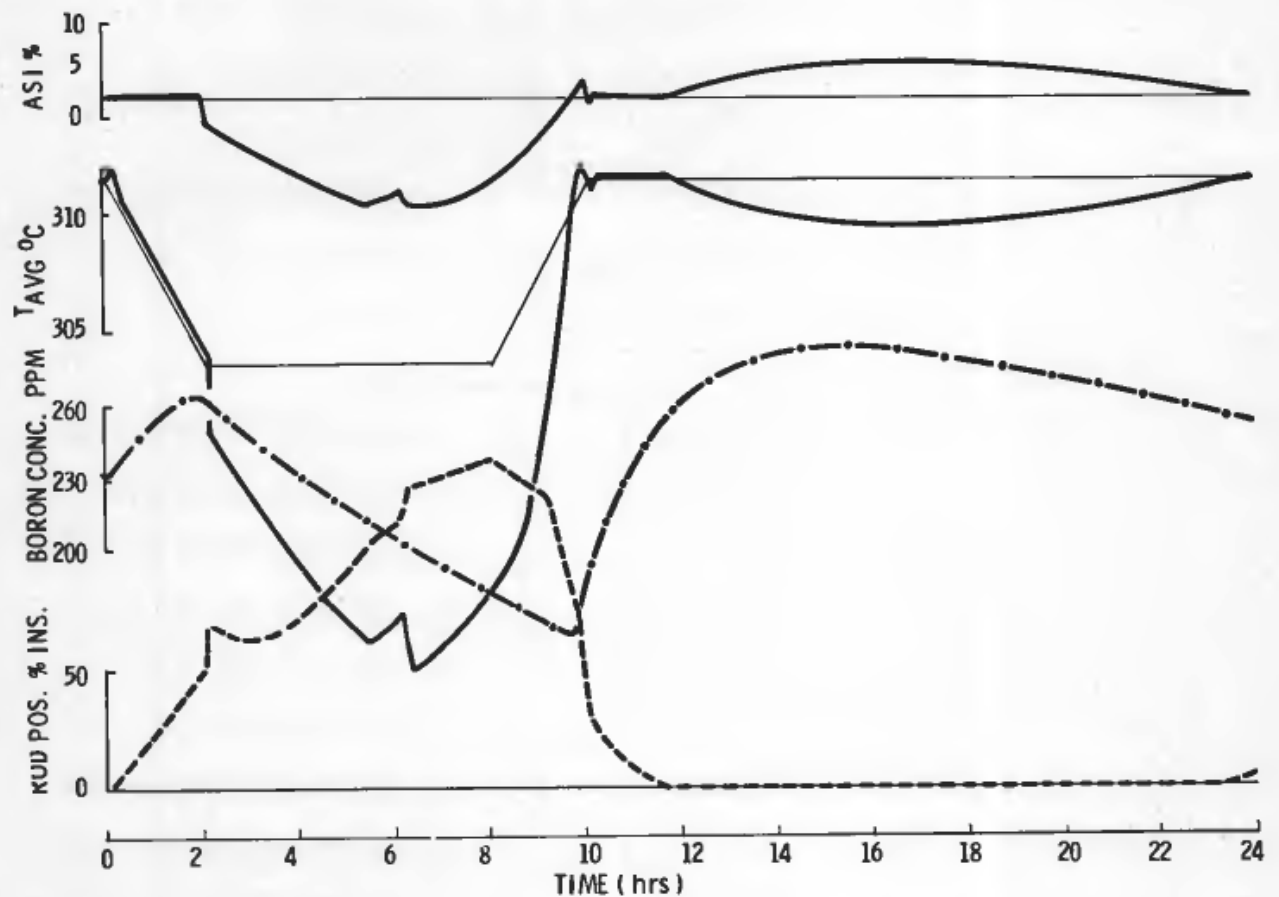

Figure 6. SVF controlled $50 \%$ load cycle.
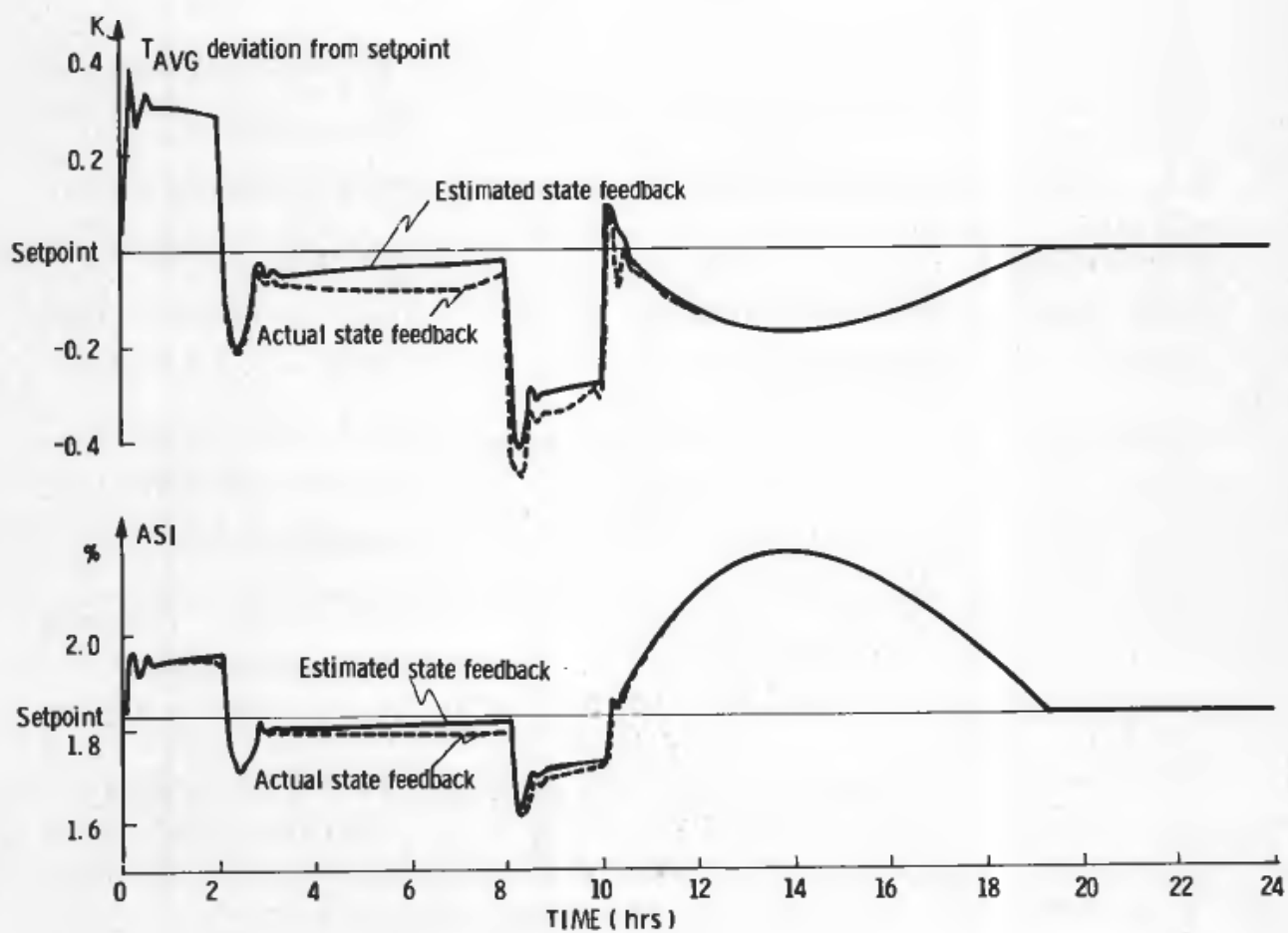

Figure 7. Comparison of SVF with feedback from estimated state and feedback from actual state. 

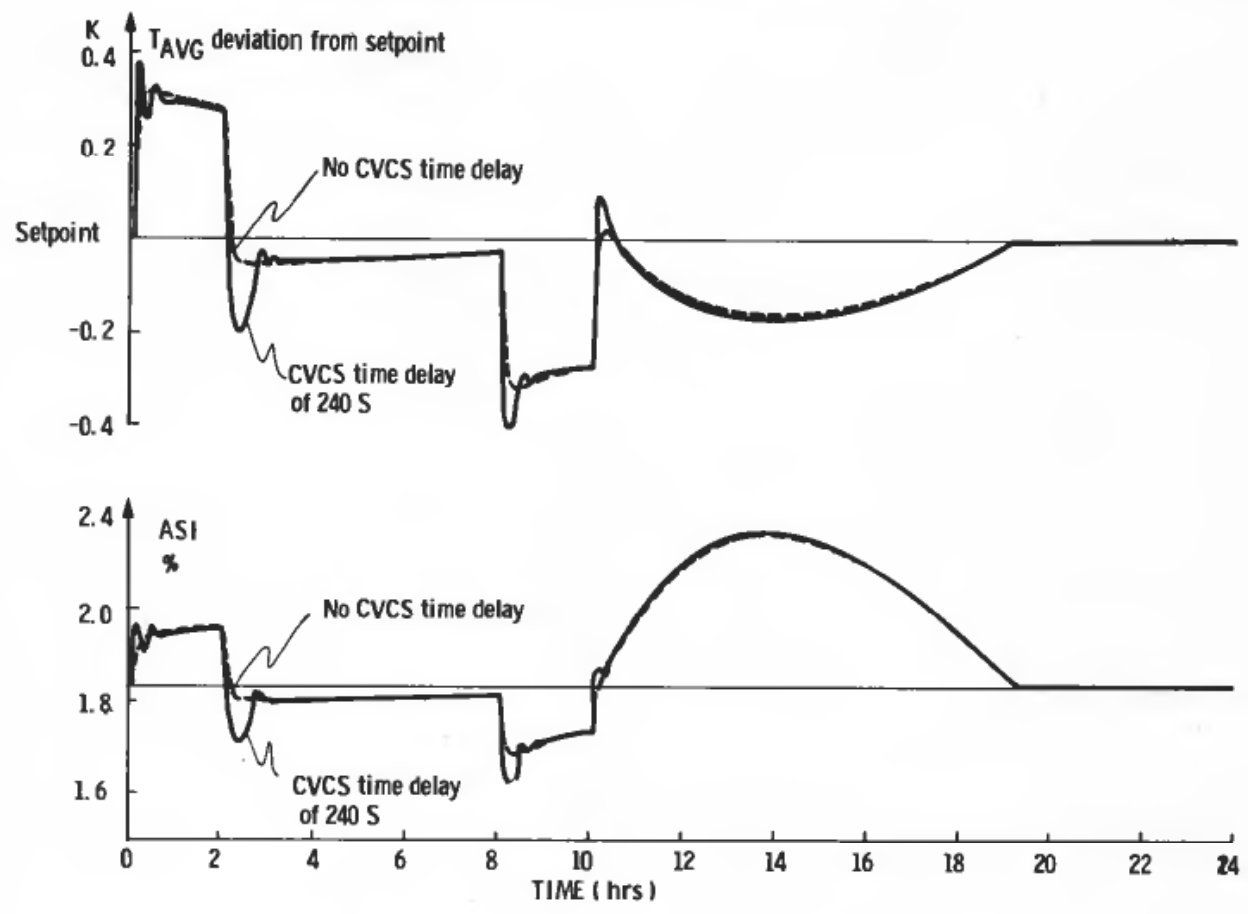

Figure 8. Comparison of SVF with and without CVCS time delay included in simulated plant.

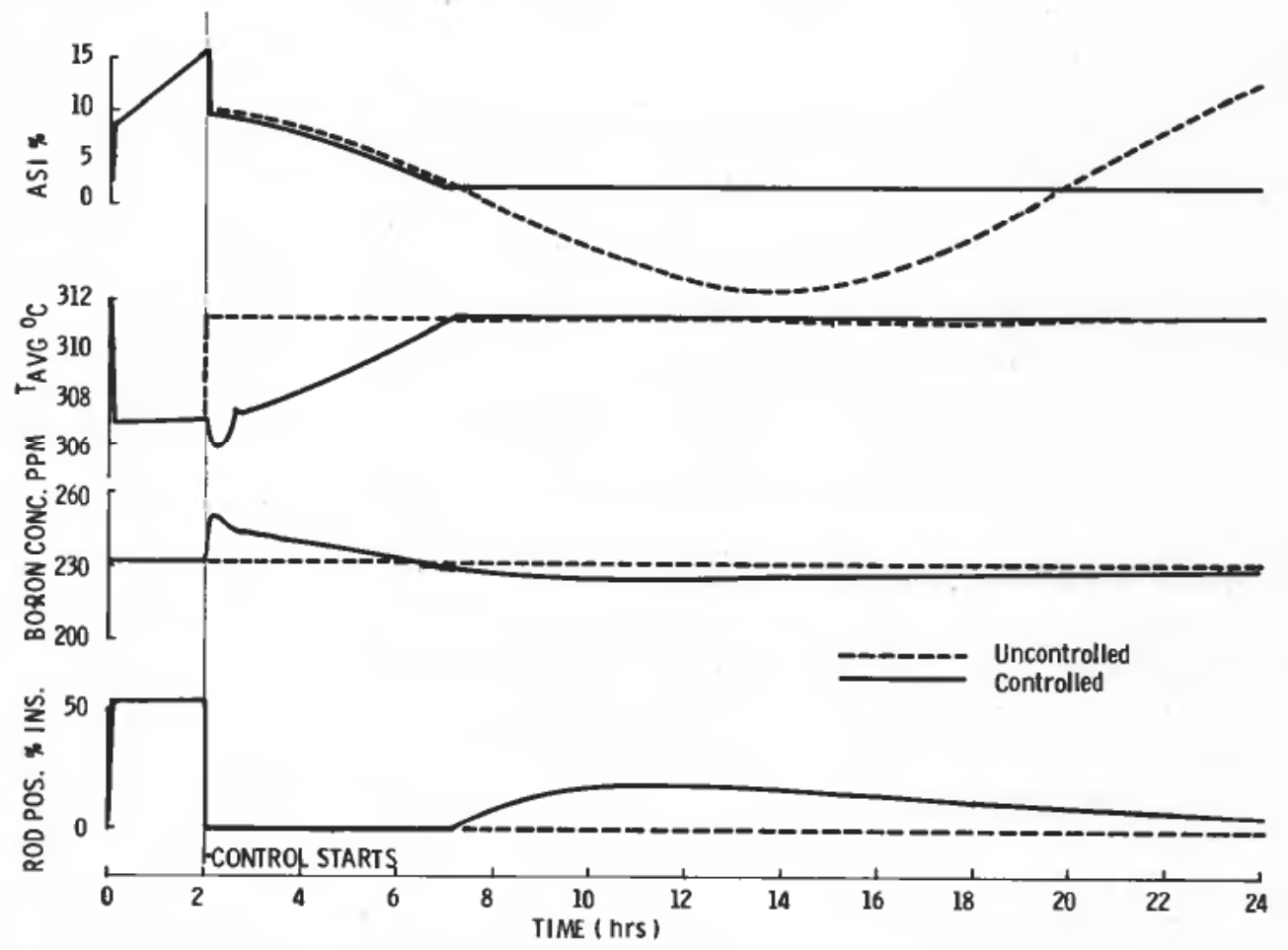

Figure 9. SVF control after disturbance. Control starts at $2 \mathrm{~h}$. 
the first control rod bank is moved in about $50 \%$ and kept there for $2 \mathrm{~h}$. Figure 9 shows the simulation result when SVF is used to control ASI and $T_{\text {avg }}$, compared to the uncontrolled core after withdrawal of the control rod bank. The uncontrolled core will be subject to xenon oscillations, unstable or with very low damping, indicated in the figure. The SVF control succeeds in controlling the ASI and the $T_{\text {avg }}$ to their setpoints $5 \mathrm{~h}$ after the control starts, and then keeping the values constant. However, as seen from the simulation results, the control inputs saturate the CEA position and force the $T_{\text {avg }}$ to drop during the first $5 \mathrm{~h}$ of control. In this period only the boron control is active, which does not improve the control performance. After the setpoints are reached, boron and CEA control act simultaneously to stabilize the core.

In summary, the simulation experiments show that the state variable feedback control combined with a turbine power disturbance feedforward control gives as good a control performance, within the limitations of the control system hardware, as can be expected from a feedback control system. Control actuator saturation, particularly boron dilution, is the most serious barrier to obtaining 'perfect' control; non-linear CEA action produces some 'non-ideal' control system behaviour as well. The forced process behaviour occurring when a control actuator is saturated leads in none of the cases considered to serious problems from the larger core control point of view, and in all cases considered the process dynamic causes recovery from saturation within an appropriate time.

As for the SVF control system performance in a more narrow sense, it was shown that:

SVF is fairly sensitive to changes in setpoints. Expansion of the feedforward control feature is expected to reduce this problem.

The Kalman state estimator converges sufficiently fast for use with the SVF control system.

Integrated output error feedback to prevent static error causes oscillations when controller saturation is encountered and during recovery from saturation; control system performance does not appear to benefit from integrated output error feedback. On the other hand, if control system setpoints are calculated using a linear process model, static error may occur.

The controller performance may easily be changed by varying the objective function weight parameters. However, the effect of weight parameter changes may not always be easy to predict, so verification of performance changes via simulation experiments is required.

\section{Multivariable frequency response (MFR)}

This section describes in some detail the application of MFR techniques to the design of an Output Feedback for a large PWR NSSS. The main task of the Control System will be to maintain two important core variables, namely average primary coolant temperature $\left(T_{\text {avg }}\right)$ and axial shape index (ASI), at their desired values during load transients.

\subsection{MFR design}

The design of MFR is conveniently formulated as a 'regulator' problem, i.e., one in which chief requirements are stability in all operating conditions, satisfactory temperature control and good ASI control, especially at high power levels, as 
opposed to a 'servo' problem, in which severe requirements are posed on the closed loop response to rapid changes in the desired values.

The desired values (or setpoints) of $T_{\text {avg }}$ and ASI, in fact, are likely either to stay constant or to vary at relatively slow rates and by small amounts from typical standard values. In this context the total power applied by the NSSS (usually the turbine power $\boldsymbol{P}_{\mathrm{t}}$ ) is recognized as the main (measurable) disturbance which affects the system.

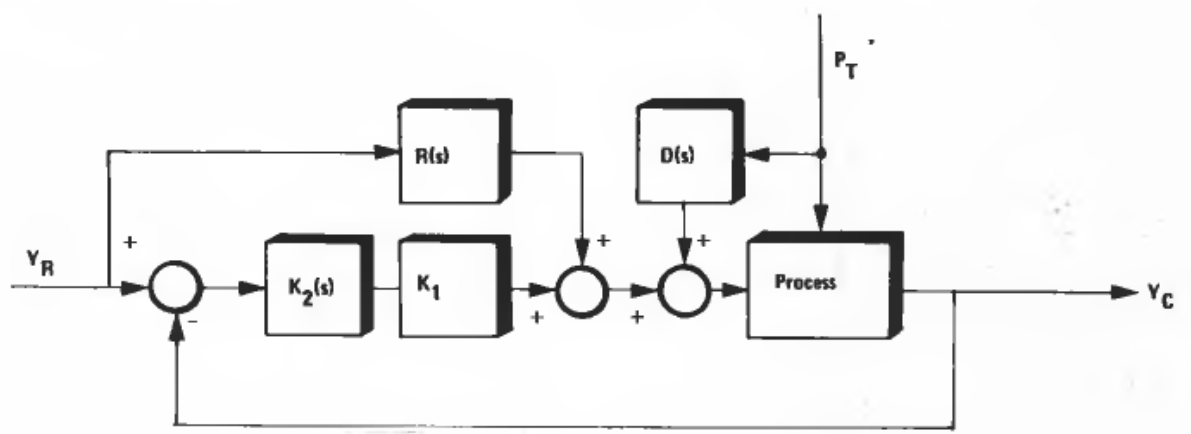

Figure 10. Scheme of the MFR regulator.

The MFR design procedure results in a control system of the form given in Fig. 10. The system consists of a feedback control strategy $K_{2}(s)$ and a compensator $K_{1}$. The idea of the compensator is to generate an almost diagonalized system for the relevant frequency range. The product of the plant and the compensator is said to be diagonal dominant, and the methods for testing the diagonal dominance is described by Rosenbrock (1974). The feedback control is then designed with traditional frequency response techniques resulting in a diagonal feedback compensator matrix $K_{2}(s)$. Feedforward control is supplemented from the setpoints $(R(s))$ and from the disturbance $(D(s))$.

The design procedure of MFR starts with a linearized model of the NSSS (described in $\S 2.1$ ) in the form

$$
\left.\begin{array}{l}
\dot{\boldsymbol{x}}=A x+B u+C_{\mathrm{x}} d \\
y=D x
\end{array}\right\}
$$

as produced by the TOPO program, and corresponding to the linear model (1) for the SVF design. The input $\boldsymbol{u}$ and output $\boldsymbol{y}$ consist of

$$
\begin{aligned}
& u_{1} \text {-control rod speed } \\
& u_{2} \text {-boron concentration at charging node } \\
& y_{1} \text {-average primary coolant temperature, } t_{\mathrm{avg}} \\
& y_{2} \text {-axial shape index, ASI }
\end{aligned}
$$

The frequency domain analysis are performed by the program VALZER.

First the transfer matrix relating the two inputs and the two outputs is calculated, giving

$$
G_{1}(s)=\frac{1}{d(s)}\left[\begin{array}{ll}
k_{11} n_{11}(s) & k_{12} n_{12}(s) \\
k_{21} n_{21}(s) & k_{22} n_{22}(s)
\end{array}\right]
$$



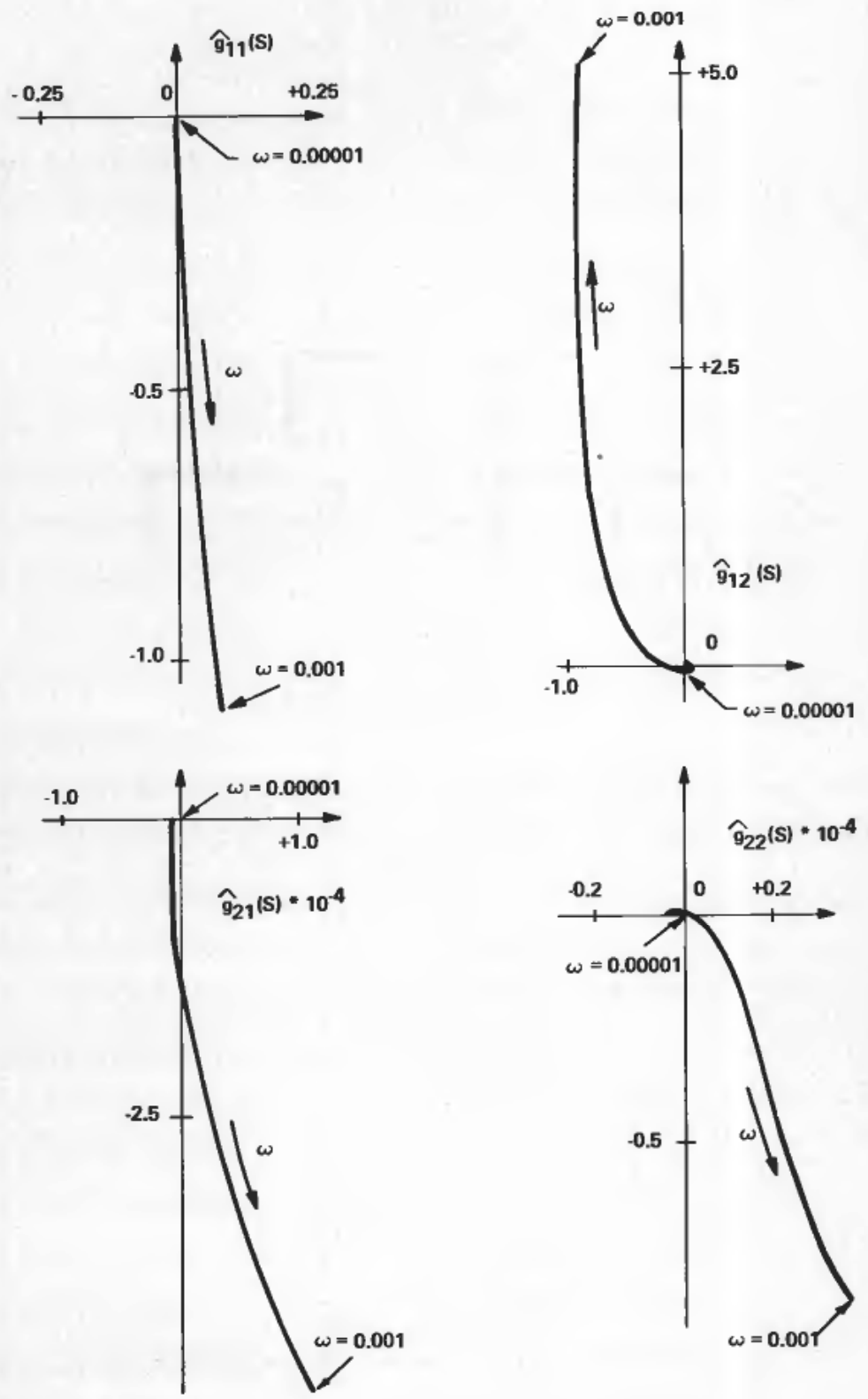

Figure 11. INA-uncompensated transfer matrix $G(s)$.

where $d(s)$ and $n_{i j}(s)$ are unimodular polynomials and the $k_{i j}$ are (gain) constants. The roots of $d(s)$ (system poles) and of $n_{i j}(s)$ (the zeros) show a pair of positive real part poles which are recognized as corresponding to the unstable axial power oscillation which is known to occur in the uncontrolled core. The zeros of $G_{1}(s)$ are all located in the left half-plane.

At this point, the time delay due to the mass transport along the charging pipes is easily accounted for by simply multiplying $G_{1}(s)$ by an irrational matrix containing 
$T_{\mathrm{d}}$, the time delay, so that the complete process will be described by the transfer matrix

$$
G(s)=G_{1}(s)\left[\begin{array}{cc}
1 & 0 \\
0 & \exp \left(-s^{T_{d}}\right)
\end{array}\right]
$$

The Inverse Nyquist Array (INA) for the uncompensated transfer matrix $G(s)$ is shown in Fig. 11. Without plotting the Gershgorin discs it is already evident that the inverse of $G(s)$ is not dominant. However, for a certain range of frequencies around $\omega=0.001, G^{-1}(j \omega)$ can be roughly approximated by:

$$
G^{-1}(j \omega) \simeq \frac{1}{j \omega}\left[\begin{array}{cc}
-1 \cdot 15 & 5 \cdot 1 \\
-5 \cdot 5 \times 10^{4} & -0.95 \times 10^{4}
\end{array}\right] \triangleq \frac{1}{j \omega} K_{1}
$$

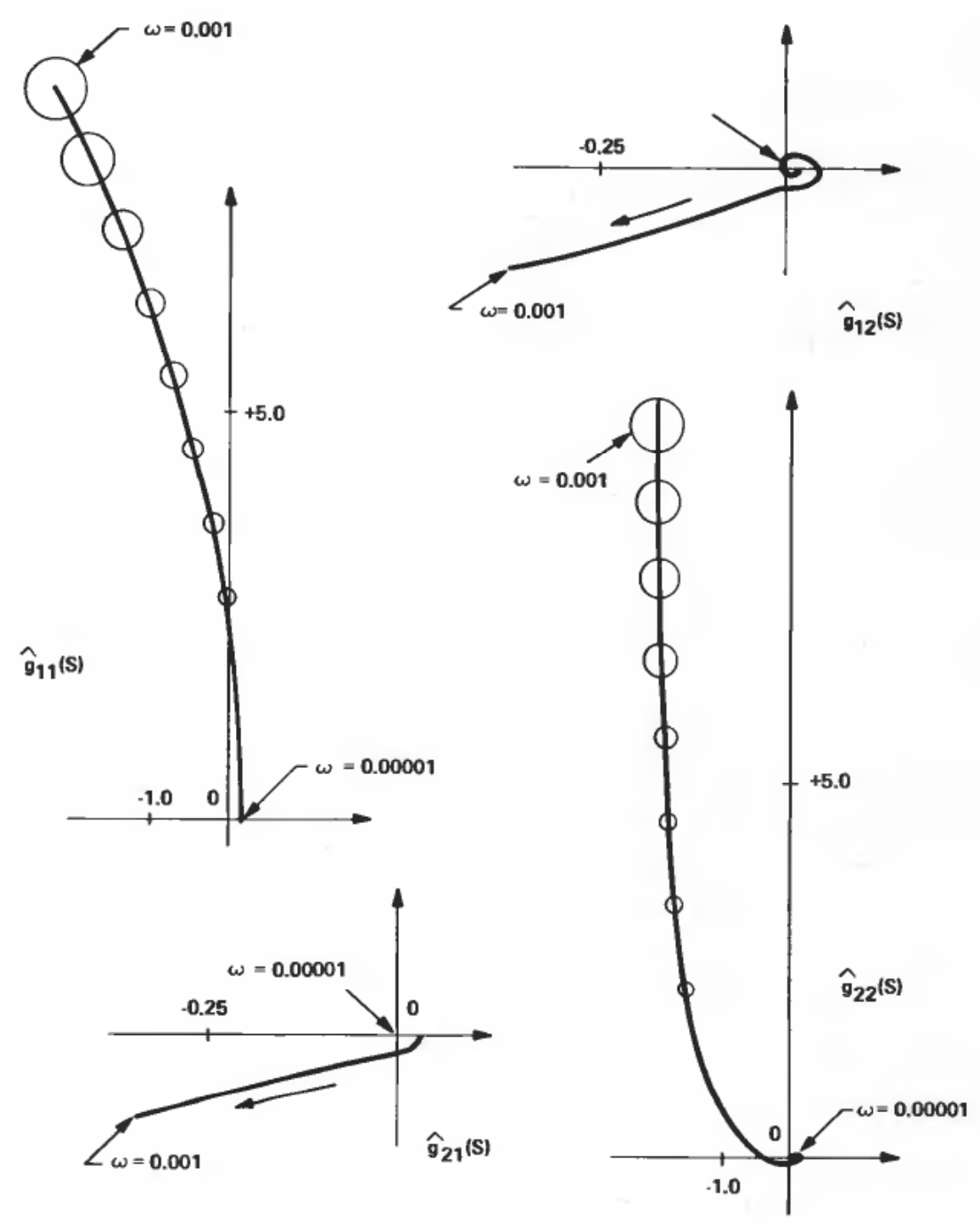

Figure 12. INA-compensated system. 

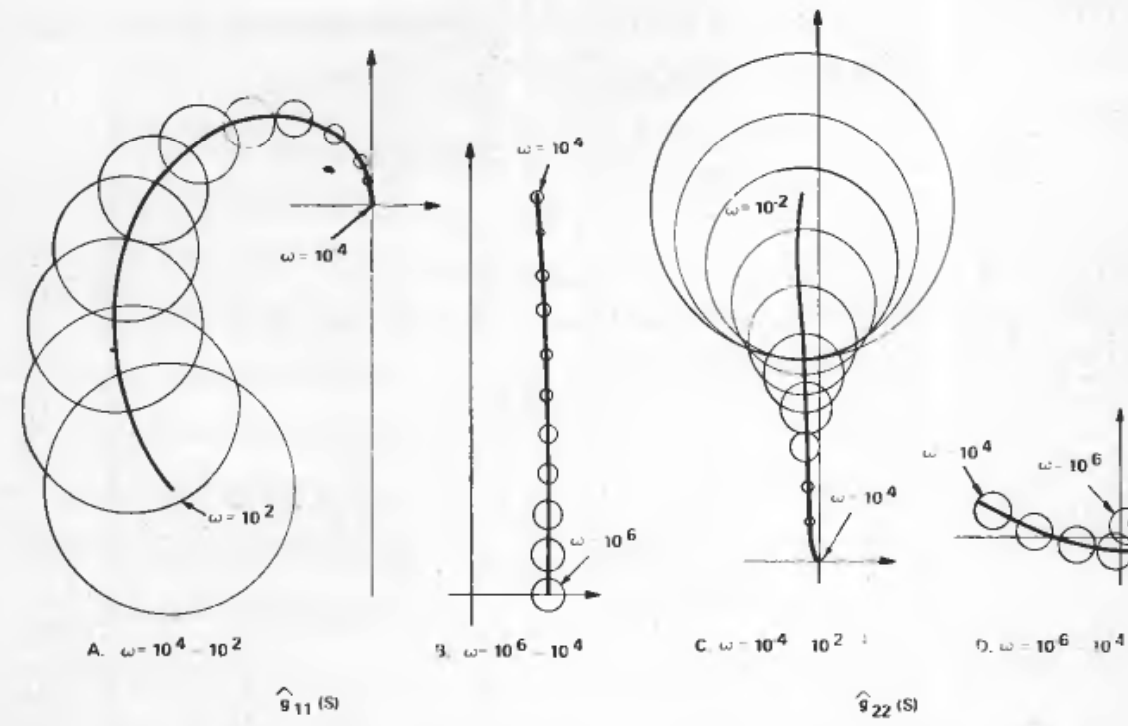

Figure 13. INA-compensated system for relevant frequency ranges.

This suggests that $Q^{-1}(s)=K_{1}^{-1} \cdot G^{-1}(s)$ should be dominant around $\omega=0.001$. This is confirmed by the INA of Fig. 12 where the Gershgorin discs are superimposed and a gain of 10 was injected in both loops. Dominance has been achieved over a wide range of frequencies (Fig. 13) although an enlargement of the Gershgorin band of $q_{22}$ around the origin (Fig. $13(d)$ ) shows that the second row is not dominant for $\omega<7 \times 10^{-5}$. Dominance below this frequency, however, is not necessary as the generalized Nyquist criterion for closed loop stability can already be used at this stage, in one of its versions. The criterion shows that the closed loop systems will be stable provided that the two loop gains satisfy the conditions $0<f_{1}<30$ and $f_{2}>0.5$. Within these limits, the loop gains may be chosen based on performance requirements. At this point, all the engineering constraints and the effect of nonlinearities should also be taken into account. Consequently, $f_{1}=30$ and $f_{2}=10$ were tentatively chosen for testing by a full non-linear simulation of the controlled system.

Prior to that, however, a dynamic compensator was designed to account for the effect of the measureable disturbance (turbine power $P_{t}$ ), in order to improve the disturbance compensation, especially at high frequencies. The stability limit $f_{1}<30$, has the effect of reducing the time response of the first loop to approximately $30 \mathrm{~min}$. Until this feedback response is felt, temperature deviations are more easily compensated by feed-forward actions. The design results in a dynamic compensator of the form

$$
R(s)=-G_{1}^{-1}(s) \cdot G_{2}(s)
$$

where the transfer matrix $G_{2}(s)$ relates the output $y$ to the disturbance $P_{t}, G_{2}(s)$ is computed by VALZER from eqn. (12). After approximations this results in a dynamic compensator

$$
R(s)=\left[\begin{array}{c}
r_{11} \cdot s \\
r_{21} \frac{\left(s-z_{1}\right)\left(s-z_{2}\right)}{\left(s-p_{1}\right)}
\end{array}\right]
$$




\subsection{MFR simulation results}

At this point, the complete MFR control system, including the feedback compensator $K_{2}$ with the tentatively selected loop gains and the feed-forward dynamic compensator $R(s)$, was submitted to extensive simulation tests with the non-linear simulator TOPO in order to study its performance in different operating conditions. From the simulation experiments some are selected in order to compare the SVF and MFR performance. Figures 14 and 15 present the MFR performance during $75 \%$ respective $60 \%$ loadcycle, as described by Fig. 3 . As may be seen from the results, only minor discrepancies are observed. The discussion of the control system behaviour is not repeated here.

The study of the MFR control system has been given an additional objective, that is the demonstration of the capability of integrating the multivariable feedback control into the existing conventional control system, in this case the Reactor Regulating System (RRS) controlling the control rod movements. This has been done by taking the dynamics of the RRS into consideration and designing an Integrated Core Control System (ICCS) which generate an input signal to the RRS, in addition to the other input signals RRS usually utilizes. The compensator for the RRS has been included into the MFR control system, and the simulator TOPO has been extended to model the conventional RRS.

A typical daily loadcycle was chosen from among several load manoeuvres which were studied to discuss the ICCS performance, that is the $50 \%$ loadcycle in Fig. 3. The simulated system response is shown in Fig. 16. It appears (not surprisingly) to suffer from the same saturation and non-linearity problems as the other control system designed. However, from an operational point of view the $T_{\text {avg }}$ deviation is
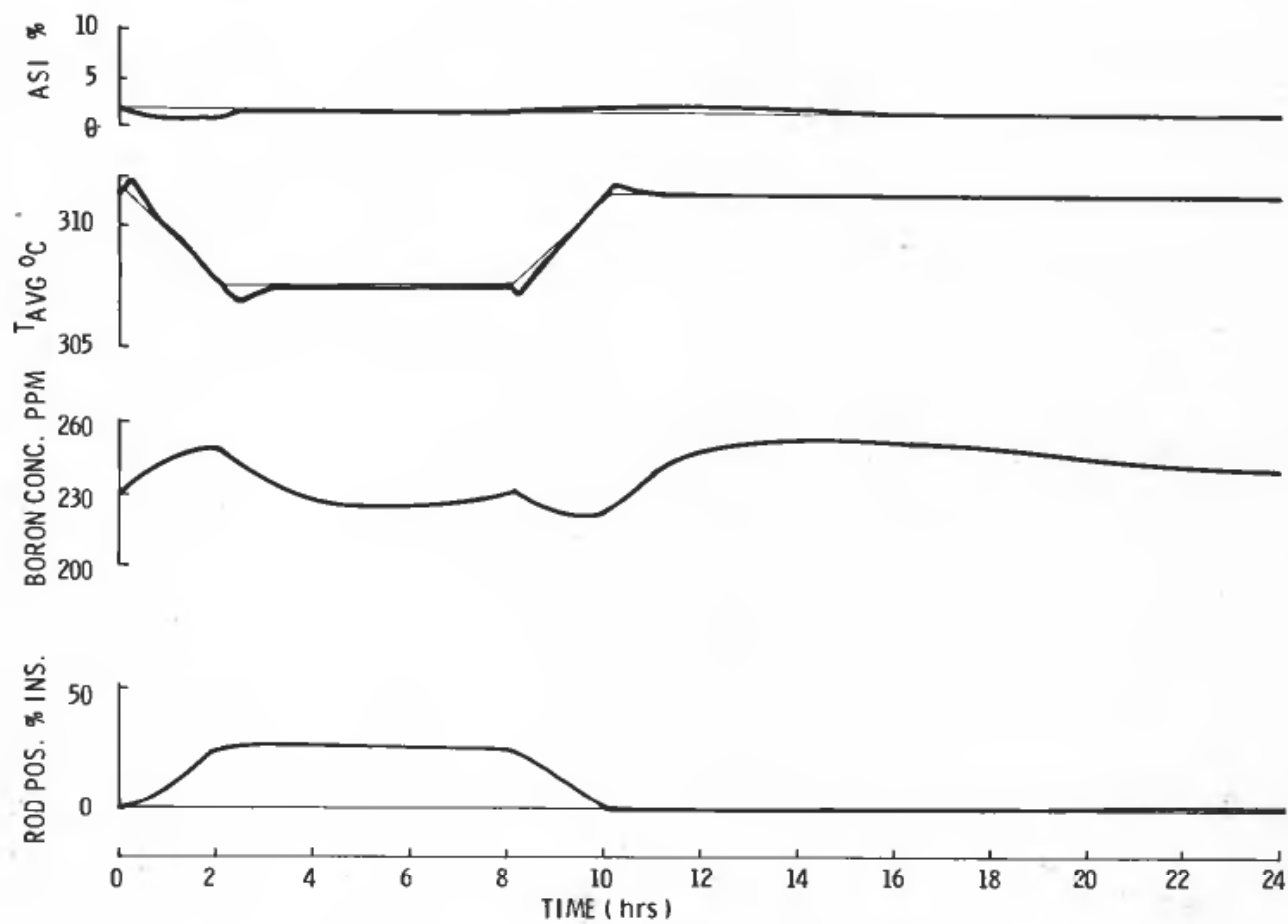

Figure 14. MFR controlled $75 \%$ load cycle. 


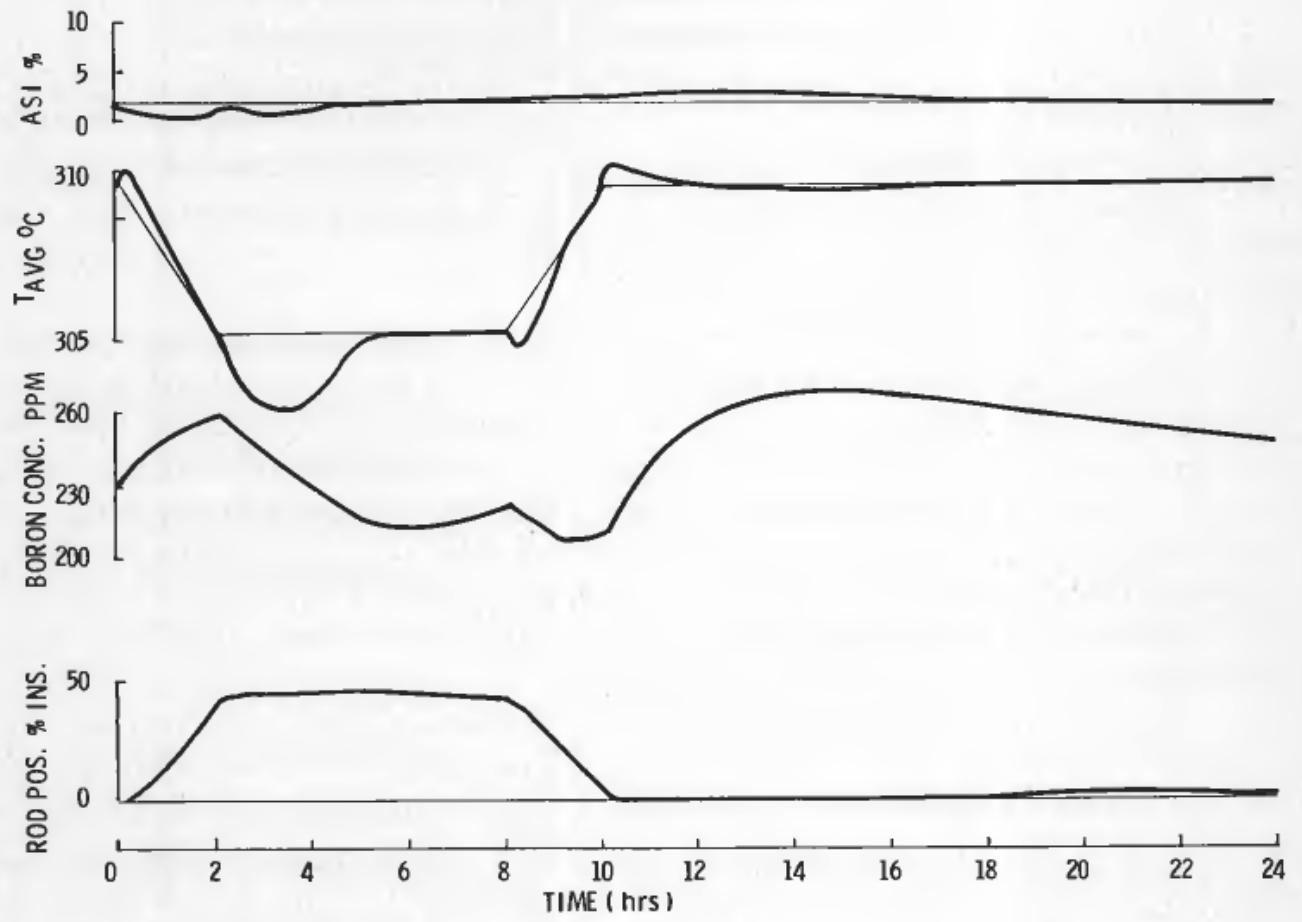

Figure 15. MFR controlled $60 \%$ load cycle.

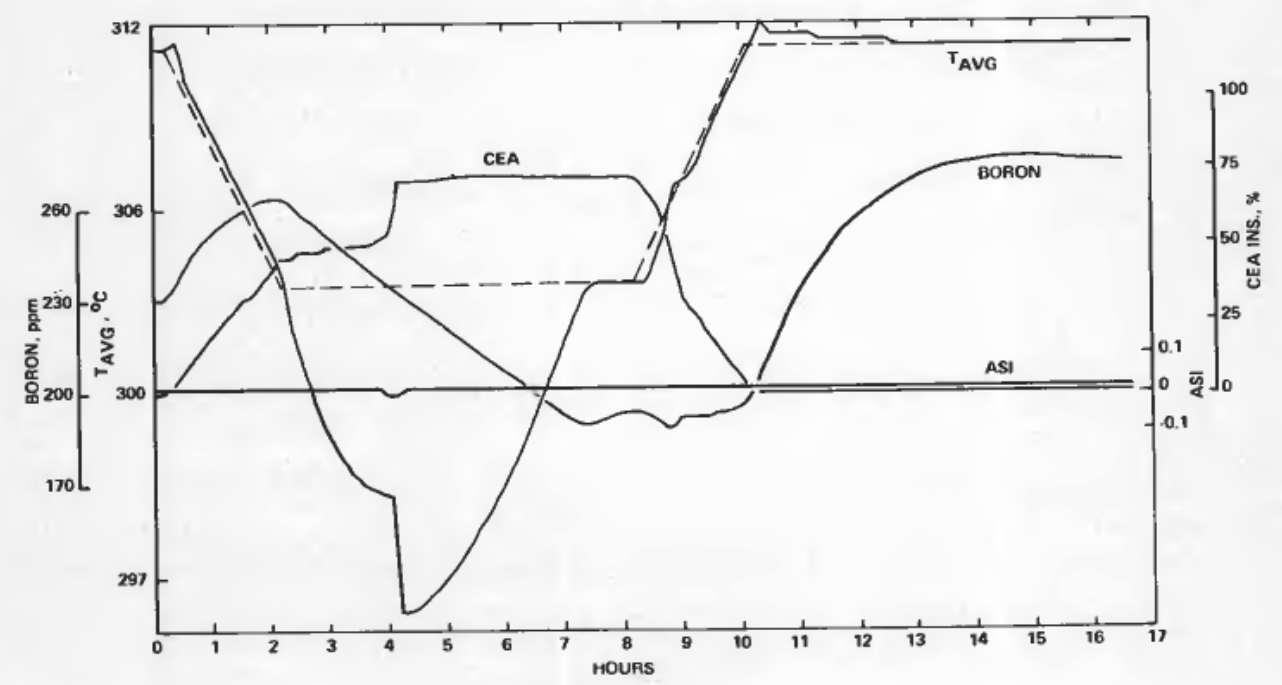

Figure 16. Daily load cycle ICCS.1 control system. 


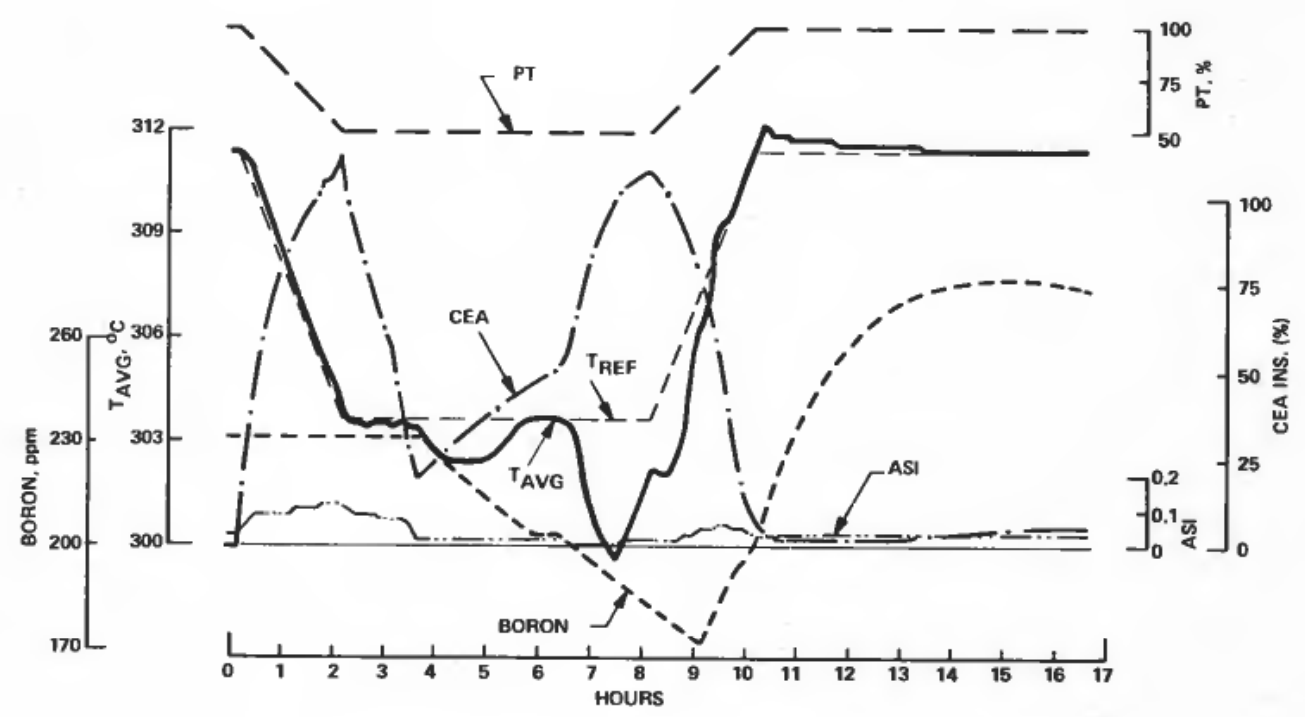

Figure 17. Daily load cycle--minimizing waste water production-AICCS.2 control system.

not regarded as serious. It may be noted that the ASI performance is improved compared to the SVF control system, and this may indicate that MFR is less sensitive to large deviations from the working (or linearization) condition.

\section{Conclusion}

The simulation experiments show that application of automatic multivariable feedback control (MFC) in the power and shape control systems of PWR's may be useful and practical. The simulations show that the power shape and the primary coolant temperature are well controlled during a loadcycle and after a disturbance, without operator intervention. In addition the MFC can reduce the waste water generation by minimizing the changes in coolant boron concentration.

Neither one of the two methods applied, State Variable Feedback (SVF) or Output Feedback by Multivariable Frequency Response Technique (MFR) has proved to be significantly better than the other one with respect to the simulated performance or with respect to computer requirements. However, the MFR method results in a simpler control algorithm compared to SVF, which includes a state estimator. The MFR method also appears somewhat less sensitive than SVF when large deviations from the linearity assumptions in the control model occur. On the other side the SVF method results in a better control (more optimal) during small disturbances. The tuning of the MFR controller to obtain more optimality has not been demonstrated.

The SVF estimator (Kalman filter) has been tested and found to be satisfactory for the state feedback control. Other use of the estimator (e.g., in a core surveillance 
system) has not been tested, but the simulation results indicate that the SVF method is applicable for state estimation even if a MFR output control is selected.

The multivariable feedback control methods are, as shown in the simulation experiments, sensitive to the control input saturation. The reason is that hard constraints cannot be satisfactorily described in the control problem formulation. This may not be considered a too serious problem, because there exist practical ways of getting around it.

The saturation problem is not expected to have serious consequences because saturation leading to temperature increase is not likely to happen: If borating saturates (the saturation limit is ten times bigger than the dilution saturation limit), then control rods are always ready to be inserted.

Both methods are based on the same linearized model of the process. The number of state variables is independent of the control design method. The linear model is designed to give a simple but sufficient description of the output variable behaviour. Starting from the linearized model, the MFR output control and the SVF state control design procedures proceed in quite different ways.

The SVF design procedure involves solutions of the optimal state feedback and the state estimation problem by proper selection of weighting matrices and solving the matrix Riccati equations for both problems. Both the feedback control law and the filter gain have to be tested by simulation experiments. Tuning of the feedback and the estimator may be laborious, because it implies repetitions of the design procedure almost from the beginning.

The MFR design procedure involves design of a compensator to diagonalize the system in the relevant frequency range. The Inverse Nyquist Array technique is suitable for this design. Traditional frequency domain techniques are used for the design of feedback and feedforward compensators. The resulting control system has to be tested by simulation. Some trial and error repetitions have to be expected for the design of feedback and feedforward compensators, however, less laborious than in the SVF design procedure.

As noted, both methods require evaluation by simulation experiments, and then potentially changes in the regulator. This is important for the initial design of the control system, and several iterations may be necessary for a satisfactory controller performance.

During the design of this particular NSSS control system, the MFR method was easier to apply. This is mainly because of the relatively difficult task of tuning the SVF controller, while MFR is more directly designed from the Nyquist and Bode plots. In addition, a sufficient phase margin for the CVCS time delay is directly introduced, while the corresponding phase margin for the SVF control must be experimentally verified with simulation.

However, as the fuel of the reactor is burning and the core parameters change, the control system has to be maintained during reactor core life. This control design procedure is expected to converge rapidly, and the conclusion is that the laborious initial design procedure is performed only once. The differences of MFR and SVF in that respect should not be overly stressed.

Both SVF and MFR require fairly sophisticated computer code libraries for the control design. In both cases the linear model order defines the computer requirements. In addition, computer capacity for the simulation and linear model generation must be present. This is likely to be the main computational task for the design. 
As a general conclusion, none of the above arguments significantly favour one approach over the other, so that the control designer is advised to use the approach that is most familiar.

Finally, some comments are made regarding the relationship between the feedback control methods presented in this paper, and the predictive deterministic optimal control methods being developed for control of nuclear reactor cores. These methods are based on highly detailed models which with great precision calculates the optimal control actions over several hours, up to $20-30 \mathrm{~h}$. At the Halden Reactor Project the MMP Multistage Mathematical Programming method has been developed and used in simulation studies on core power distribution optimization (MIC 1980, Vol. 1, No. 2), and presently the method of MCCS (Multilevel Core Control System) is being tested. The second method is based on hierarchical optimization and control, and is described by Beraha (1981).

The predictive optimal control methods are in their present stage of development not well suited for on-line applications, because of the heavy computational burden of the detailed models. On the other side, the multivariable feedback control methods may easily be implemented on-line after the feedback law once is calculated. However, the MCCS method includes a feedback control method as one of its subsystems, and may be expected to be further developed for on-line applications.

In general, the main drawback of the MFR and SVF methods is the limited number of controllers and controlled outputs. For the nuclear power plant which is described in this paper and which is fundament for the MFR/SVF development, this is not a serious limitation because it reflects the operational strategy of the plant. But in general a higher number of controllers may be used (several control rod banks) to control a greater number of outputs. At the Halden Reactor Project one is evaluating the possibility of utilizing a combination of the predictive and the feedback control methods: Using MMP or MCCS one calculates the optimal control during a predictable (or planned) plant disturbance over the next hours. During the application of this detailed control, the multivariable feedback control method is utilized to compensate for the non-predictable disturbances, using the precalculated values as setpoints.

\section{ACKNOWLEDGEMENTS}

This paper is a presentation of a joint project between Ente Nazionale per l'Energia Elettrica (ENEL), Italy, Combustion Engineering, Inc., USA, and the OECD Halden Reactor Project, Norway. The application of the multivariable frequency domain technique has been performed by Mr. Teresio Busi, ENEL.

\section{REFERENCES}

BLOMSNES, B., and KARPPINEN, J. (1976). An application of optimization methods to spatial control of nuclear reactor cores, in International Symposium on new Trends in System Analysis, 13th-17th December, Rocquencourt, France.

BollacasA, D., and BusI, T. (1981). Multivariable feedback control applications in C-E high power density PWRs, in International Topical Meeting on Advances in Mathematical Methods for the Solution of Nuclear Engineering Problems, April 27th-29th. München, Germany.

HAUGSET, K., and LEIKKONEN, I. (1980). Nuclear reactor control by multistage mathematical programming. Modeling, Identification and Control, 1, 119-133. 
Karppinen, J., Versluis, R. M., and Blomsnes, B. (1979). Core control optimization for scheduled load changes in large pressurized water reactors. Nucl. Sci. Eng., 71, 1-17. Rosenbrock, H. H. (1971). Computer-Aided Control System Design (Academic Press).

Beraha, D., and KarpPInen, J. (1981). Power distribution control by hierarchical optimisation techniques, in International Topical Meeting on Adiances in Mathematical Methods for the Solution of Nuclear Engineering Problems, April 27th-29th, München, Germany. 